\title{
Review Article \\ Dapagliflozin in Patients with Chronic Heart Failure: A Systematic Review and Meta-Analysis
}

\author{
Ru-ping Cai $(\mathbb{D}$, Yu-li Xu, and Qiang Su \\ Department of Cardiology, Affiliated Hospital of Guilin Medical University, Guilin, China \\ Correspondence should be addressed to Qiang Su; drsuqiang@163.com
}

Received 18 December 2020; Revised 3 March 2021; Accepted 14 March 2021; Published 31 March 2021

Academic Editor: Frank Dini

Copyright (C) $2021 \mathrm{Ru}$-ping Cai et al. This is an open access article distributed under the Creative Commons Attribution License, which permits unrestricted use, distribution, and reproduction in any medium, provided the original work is properly cited.

\begin{abstract}
Sodium-glucose cotransporter-2 (SGLT2) inhibitors represent newly developed oral antidiabetic drugs that are practiced for type 2 diabetes mellitus management and may decrease the risk of the first hospitalization in heart failure. The activity of SGLT2 inhibitors is not related to glucose, and the effectiveness and safety of SGLT2 inhibitors in individuals with chronic heart failure (CHF) remain unclear. We systematically retrieved PubMed, Cochrane Library, Embase, NCKI, VIP, Wanfang Data, and ClinicalTrials.gov records to identify eligible trials. The primary endpoints were cardiovascular death/hospitalization for heart failure (CV death/HHF), cardiovascular death, and hospitalization for heart failure. Secondary endpoints included hypoglycemia, volume depletion, urinary tract infection, left ventricular ejection fraction (LVEF), and NT-proBNP. Nine randomized controlled clinical trials were included. Dapagliflozin was reported to significantly decrease CV death/HHF (relative risk (RR): 0.75 ; 95\% confidence interval (CI): 0.68-0.84), CV death (RR: 0.80; 95\% CI: 0.68-0.93), and HHF (RR: 0.72; 95\% CI: 0.63-0.83). There was no effect on hypoglycemia (RR: 0.69; 95\% CI: 0.34-1.40), volume depletion (RR: 1.17; 95\% CI: 0.97-1.41), urinary tract infection (RR: 0.82; 95\% CI: 0.43-1.57), LVEF (WMD: 0.53; 95\% CI: -4.04-5.09), or NT-proBNP (SMD: -0.66 ; 95\% CI: $-1.42-0.10$ ). The risk of CV death/HHF, CV death, and HHF was lower among patients receiving dapagliflozin than patients receiving placebo.
\end{abstract}

\section{Introduction}

According to ESC guidelines, $\mathrm{HF}$ is a clinical syndrome characterized by typical symptoms (e.g., breathlessness, ankle swelling, and fatigue) that may be accompanied by signs (e.g., elevated jugular venous pressure, pulmonary crackles, and peripheral oedema) caused by a structural and/ or functional cardiac abnormality, resulting in a reduced cardiac output and/or elevated intracardiac pressures at rest or during stress [1]. Despite advances in medicine, chronic heart failure, as a fatal disease, has always been a challenge for healthcare providers. Globally, around 64 million people suffer from CHF (half of whom have reduced ejection fraction). CHF is a chronic progressive disease, and $50 \%$ of patients die within five years after diagnosis [2]. CHF is the major cause of hospitalization for people over 65 years of age and is correlated with significant healthcare and economic burden on nations globally. Due to the use of aldosterone receptor antagonists, angiotensin-converting enzyme inhibitors (ACEIs), $\beta$-blockers, and angiotensin receptor blockers (ARBs), CHF evolved from a fatal disease to a severe disease that needs long-term team management [3]. Results of the recently conducted clinical studies have shown that sodium-glucose cotransporter-2 (SGLT2) inhibitors can reduce the risk of CHF in individuals having type 2 diabetes mellitus (T2DM) [4-9]. Recent clinical trials have shown that the SGLT2 inhibitor dapagliflozin can reduce the cardiovascular death/hospitalization for heart failure $(\mathrm{CV}$ death/HHF) in patients with CHF, whether or not they have T2DM [10-12]. Recently, the FDA authorized dapagliflozin for adult patients with HFrEF (with or without T2DM) to reduce the risk of cardiovascular deaths and hospitalization due to HF and associated disorders. This suggests that dapagliflozin may become a cornerstone and a candidate for the treatment of HF, in addition to angiotensin-converting enzyme inhibitors (ACEIs), angiotensin receptor/neprilysin inhibitors (ARNIs), beta-blockers, and mineralocorticoid receptor antagonists (MRAs). Although some clinical trials 
have demonstrated the effect of dapagliflozin in avoiding $\mathrm{HF}$, its efficacy and clinical application remain controversial and debatable, and there is no standardized clinical guideline for reference. Accordingly, we used the Cochrane systematic review methodology to objectively appraise studies that analyzed dapagliflozin in preventing and treating $\mathrm{CHF}$, intending to provide a scientific basis for clinical use.

\section{Methods}

2.1. Search Strategy. We systematically retrieved records from PubMed, Cochrane Library, Embase, NCKI, VIP, Wanfang Data, and ClinicalTrials.gov that were published before November 30, 2020, which contained one or more of the following keywords: dapagliflozin; Forxiga; Chronic Heart Failure; Myocardial Failure; Right-Sided; Systolic Heart Failure; Sodium-Glucose Transporter 2 Inhibitors; (2S, 3R, 4R, 5S, 6R)-2-(4-chloro-3-(4-ethoxybenzyl)phenyl)6-(hydroxymethyl)tetrahydro-2H-pyran-3,4,5-triol/BMS-

512148; Heart Decompensation; Left-Sided; Heart Failure.

No language restrictions were imposed. Besides, references from retained articles were manually searched to find other potentially pertinent data. The protocol was registered with PROSPERO (CRD42020215216).

\subsection{Study Inclusion and Exclusion Criteria}

\section{Inclusion criteria:}

All studies are randomized controlled trials (RCTs). The included patients conform to the following criteria: evidence of structural heart disease and manifestation of circulating congestion, age $\geq 18$ years old, ejection fraction $\leq 50 \%$, New York Heart Association (NYHA) class $\geq \mathrm{I}$, and NT-proBNP $\geq 600$ pg per milliliter (or $\geq 400 \mathrm{pg}$ per milliliter if they had been hospitalized for heart failure within the previous 12 months).

Exclusion criteria:

Observational studies, diagnostic studies, and animal experiments were excluded, type 1 diabetes mellitus, estimated glomerular filtration rate (eGFR) $<30 \mathrm{ml} /$ $\mathrm{min} / 1.73 \mathrm{~m}^{2}$, systolic blood pressure $<95 \mathrm{~mm} \mathrm{Hg}$, acute or previous myocardial infarction, moderate-to-severe liver, and kidney dysfunction.

All articles were independently reviewed using predefined inclusion and exclusion criteria. Initial inclusion/exclusion of articles was based on titles and abstracts, followed by full-text review in cases of uncertainty. Studies with a follow-up time of $<12$ weeks were excluded, as well as those that did not report any events of interest. In cases where several publications were based on a single study, we selected the RCT that included the longest follow-up time. Any discrepancies regarding inclusion or exclusion were settled by discussion. If any doubts remained, the third investigator (Qiang $\mathrm{Su}$ ) made the final decision.

2.3. Data Extraction. Two researchers (Cai Ru-ping and $\mathrm{Xu}$ $\mathrm{Yu}$-li) used standardized forms to collect data in duplicate.
In cases of discrepancy, all authors discussed the results and established a consensus. The primary endpoints were acquired from the original published manuscript: cardiovascular death/hospitalization for heart failure (CV death/ HHF), cardiovascular death, and hospitalization for heart failure; secondary endpoints: hypoglycemia, volume depletion, urinary tract infection, left ventricular ejection fraction (LVEF), and NT-proBNP. For each study, we searched the registration number, the type of the study, the data source of the primary endpoint, the drugs received by the control group, the follow-up time, and the dose (mg) of dapagliflozin. For the experimental group and control group in each study, we collected baseline characteristics (Table 1).

2.4. Quality Assessment. The consistency of the RCTs was measured using the prejudice risk evaluation method described in Cochrane Handbook 5.1.0 [19], and the following criteria have been evaluated: random sequence generation, allocation concealment, personnel, patient blindness, incomplete outcome data, selective reporting, the blindness of outcomes' assessment, and other biases. Studies were determined to have a strong degree of high, low, or unclear, based on the above 7 objects.

2.5. Statistical Analysis. Meta-analysis was implemented using RevMan 5.3 software. Relative risk (RR) was used for the analysis of efficacy, while each effect size was denoted as a $95 \%$ confidence interval (CI). A $p$ value was used for finding out heterogeneity between studies, and $p<0.1$ represented a significant degree of heterogeneity. $I^{2}>75 \%, I^{2}>50 \%$, and $I^{2}>25 \%$ represented high, moderate, and low heterogeneity between studies, respectively. Where there was statistical homogeneity between the studies $\left(p>0.1, \mathrm{I}^{2}<50 \%\right)$, the fixed-effect model was used; else, the model of random effects was adopted. We will have subgroups about the effect of heart function on results.

\section{Results}

3.1. Search Results. Through electronic retrieval, we identified and reviewed 1021 studies from the aforementioned sources as shown in Figure 1. The main reasons for their exclusion included the unavailable data (34 cases), duplicate data (8 cases), not having control group ( 7 cases), and short duration (3 cases). Overall, 9 studies [10-18] were included representing a total of 6278 patients (randomly divided into 3125 patients in the group of dapagliflozin and 3153 patients in the control group). 1108 patients with $\mathrm{CV}$ death/HHF, 592 cardiovascular deaths, 710 hospitalization for heart failure, 35 hypoglycemia, 402 cases of volume depletion, 38 patients with urinary tract infections, $602 \mathrm{LVEF}$, and 410 NT-proBNP were reported (in total).

\subsection{Research Characteristics and Risk-of-Bias Assessment.} The trials which were included in the study were published before May 1, 2020. The duration varies from 12 weeks to 4 years. Baseline characteristics by $\mathrm{CHF}$ patients are summarized in Table 1, which also provides detailed 


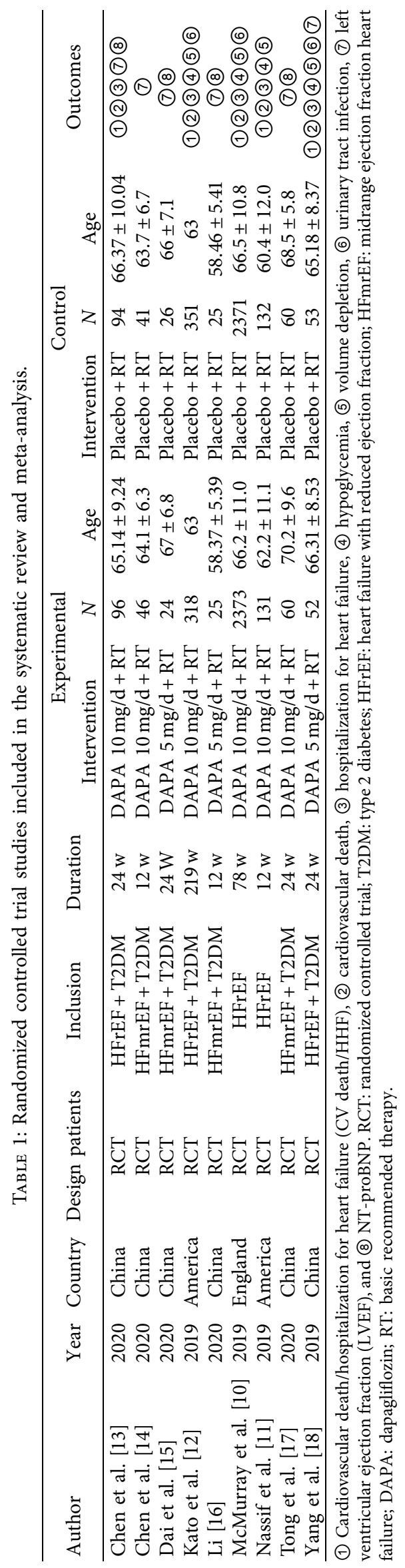




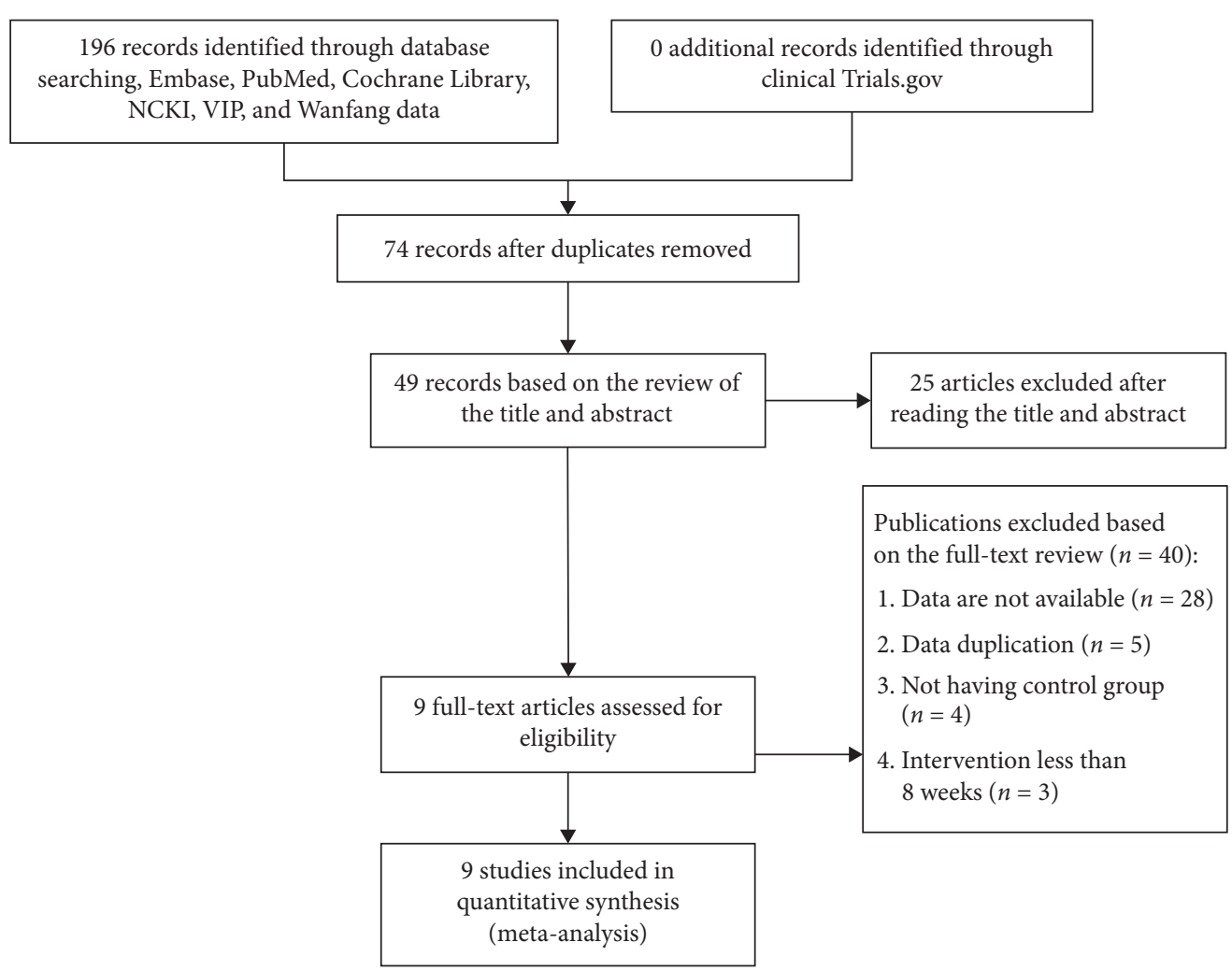

FIgURE 1: Flowchart of the study selection.

information about the included studies. Six studies $[10-13,15,17]$ used computer randomization, and 3 studies $[14,16,18]$ did not use computer randomization resulting in high risks. The overall possibility of bias in the selected reports and other biases was low, and the incomplete outcome data were unclear in a few studies that were included. Detailed information on quality assessment is shown in Figure 2.

\subsection{Meta-Analysis}

3.3.1. The Effect of Dapagliflozin on Cardiovascular Death/ Hospitalization for Heart Failure. Five studies [10-13, 18] reported $\mathrm{CV}$ death/HHF, of which 473 patients received dapagliflozin and 635 patients received a placebo. No statistical heterogeneity among the studies existed $\left(I^{2}=0 \%\right.$; $p=0.46)$. Using a model of random effects, we demonstrated that dapagliflozin highly decreased the prevalence of CV death/HHF (RR: 0.75, 95\% CI: 0.68-0.84, and $p<0.00001$ ) (Figure 3(a)).

3.3.2. The Effect of Dapagliflozin on Cardiovascular Death. Five studies [10-13,18] reported cardiovascular deaths, of which 261 patients received dapagliflozin and 331 patients received a placebo. There was no statistical heterogeneity between the studies $\left(I^{2}=0 \% ; p=0.75\right)$, and using a randomeffect model, we demonstrated that dapagliflozin significantly reduced cardiovascular deaths (RR: 0.80, 95\% CI: 0.68-0.93, and $p=0.004$ ) (Figure 3(b)).
3.3.3. The Effect of Dapagliflozin on Hospitalization for Heart Failure. Five studies $[10-13,18]$ reported hospitalization for heart failure, of which 295 patients received dapagliflozin and 415 patients received a placebo. There was no statistical heterogeneity between the studies $\left(I^{2}=0 \% ; p=0.44\right)$, and using a random-effect model, we demonstrated that dapagliflozin significantly reduced hospitalization for heart failure (RR: $0.72,95 \%$ CI: $0.63-0.83$ ), and $p<0.00001$ ) (Figure 3(c)).

3.3.4. The Effect of Dapagliflozin on Hypoglycemia, Volume Depletion, Urinary Tract Infections, Left Ventricular Ejection Fraction, and NT-proBNP. Overall, 35 cases of hypoglycemia were stated across the studies, as well as 402 cases of volume depletion and 38 cases of urinary tract infections. Meta-analysis using a random-effect model revealed that dapagliflozin did not decrease the occurrence of hypoglycemia (RR: $0.69,95 \%$ CI: $0.34-1.40$, and $p=0.3$ ) (Figure 4(a)), volume depletion (RR: 1.17, 95\% CI: 0.97-1.41, and $p=0.11$ ) (Figure 4(b)), and urinary tract infection (RR: 0.82, 95\% CI: $0.43-1.57$, and $p=0.55$ ) (Figure 4(c)). LVEF (WMD: $0.53,95 \%$ CI: $-4.04-5.09$, and $p=0.82$ ) (Figure 5(a)) and NT-proBNP (SMD: -0.66 , 95\% CI: $-1.42-0.10$, and $p=0.09$ ) (Figure $5(\mathrm{~b})$ ) were not affected by dapagliflozin.

3.4. Subgroup Analysis about the Effect of Heart Function on Results. We performed a subgroup analysis based on the NYHA class, and 7 studies $[10,11,13,15-18]$ reported 


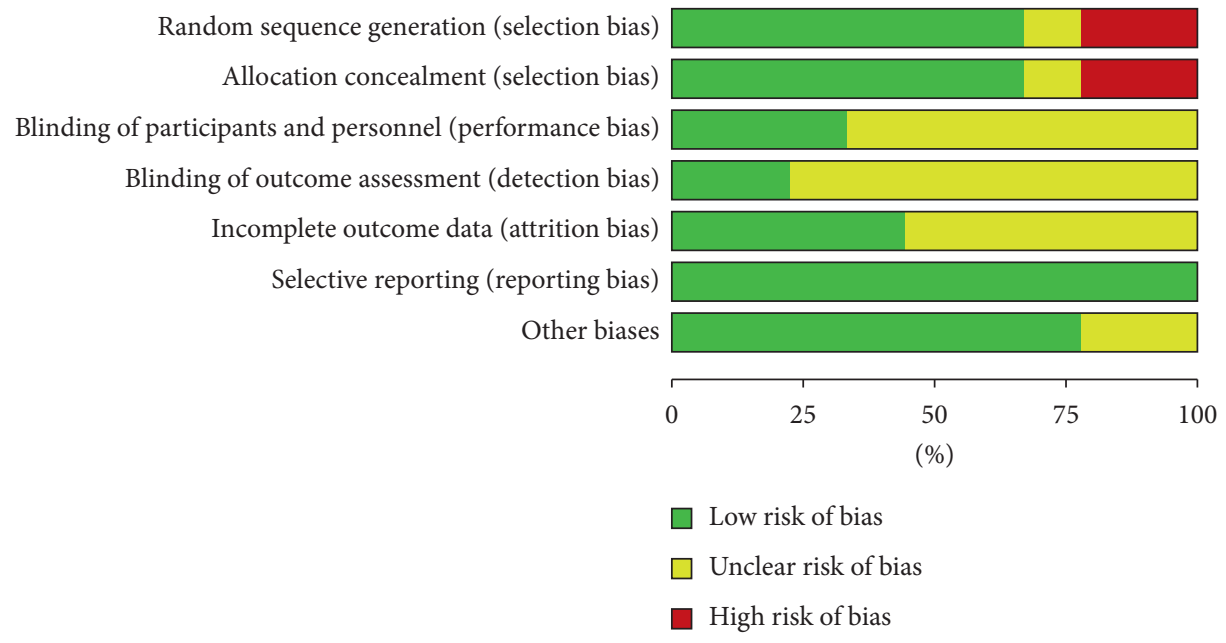

(a)

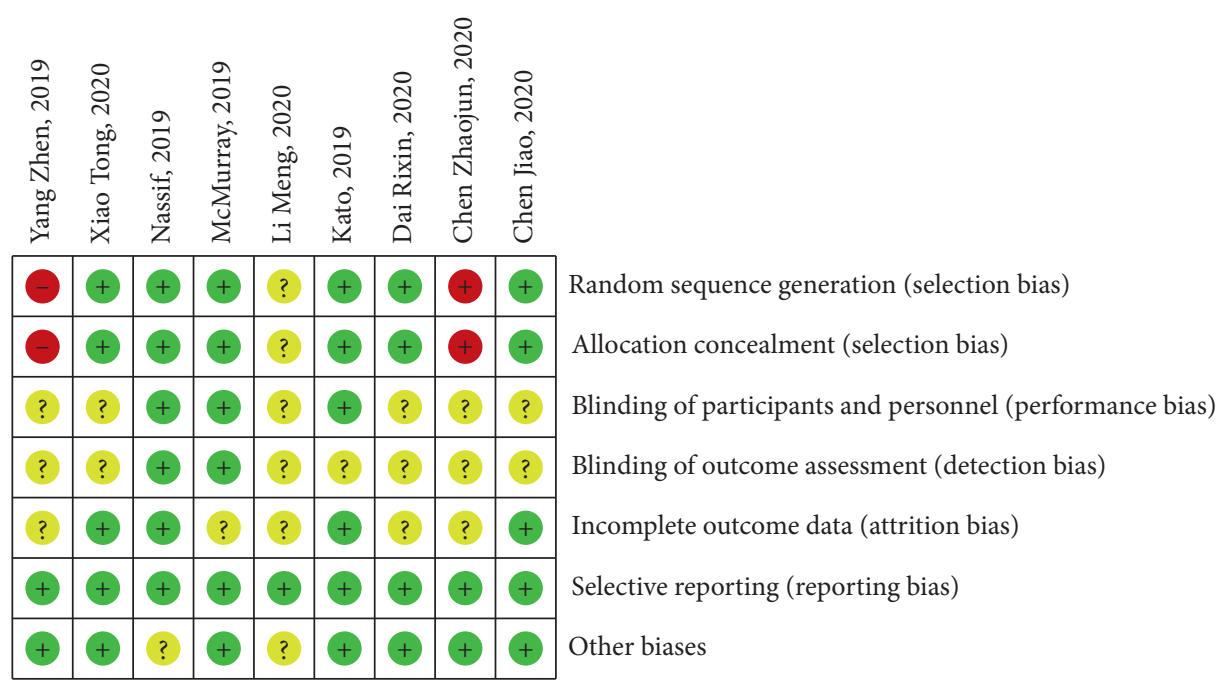

(b)

FiguRE 2: (a) "Risk of bias" graph: review authors' judgements about each risk of bias item presented as percentages across all included studies. (b) "Risk of bias" summary: review authors' judgements about each risk of bias item for each included study.

relevant data. No statistical heterogeneity was reported between studies $\left(I^{2}=0 ; p=0.74\right)$, and when a model of random effects was employed for analysis, we found that difference among the three groups was not statistically significant (RR: 1.00, 95\% CI: 0.97-1.04, and $p=0.78$ ) (Figure 6). The positive effect of dapagliflozin on patients with HF may not be related to the degree of HF; therefore, dapagliflozin is suitable for patients with mild or severe HF.

3.5. Subgroup Analysis about HFrEF and HFmrEF. We performed a subgroup analysis based on HFrEF and HFmrEF, and 8 studies $[10,11,13-18]$ reported relevant data. No statistical heterogeneity was reported between studies $\left(I^{2}=0 ; p=0.39\right)$, and when a model of random effects was employed for analysis, we found that difference among the three groups was not statistically significant (WMD: 0.37, 95\% CI: $-0.47-1.21$, and $p=0.39$ ) (Figure 7). The positive effect of dapagliflozin on patients with HF may not be related to the left ventricular ejection fraction; therefore, dapagliflozin is suitable for patients with HFrEF and HFmrEF.

3.6. Sensitivity Analyses and Reporting Bias. After excluding 2 studies [13, 15], we found that dapagliflozin can significantly improve NT-proBNP (RR: -0.45 , 95\% CI: -0.89 , -0.02 , and $p=0.04)$ and heterogeneity $(p>0.1)$. It shows that these two studies $[13,15]$ are sources of heterogeneity. The funnel chart was almost symmetrical, and the reporting bias of the studies was relatively small (Figure 8).

\section{Discussion}

SGLT2 inhibitors, which act independently of insulin, selectively inhibit SGLT2 in the kidney and block the reabsorption of glucose, thereby lowering blood glucose levels. This process does not depend on insulin resistance and $\beta$-cell function [20]. Recent studies have shown that in addition to lowering blood glucose, the SGLT2 inhibitor dapagliflozin 


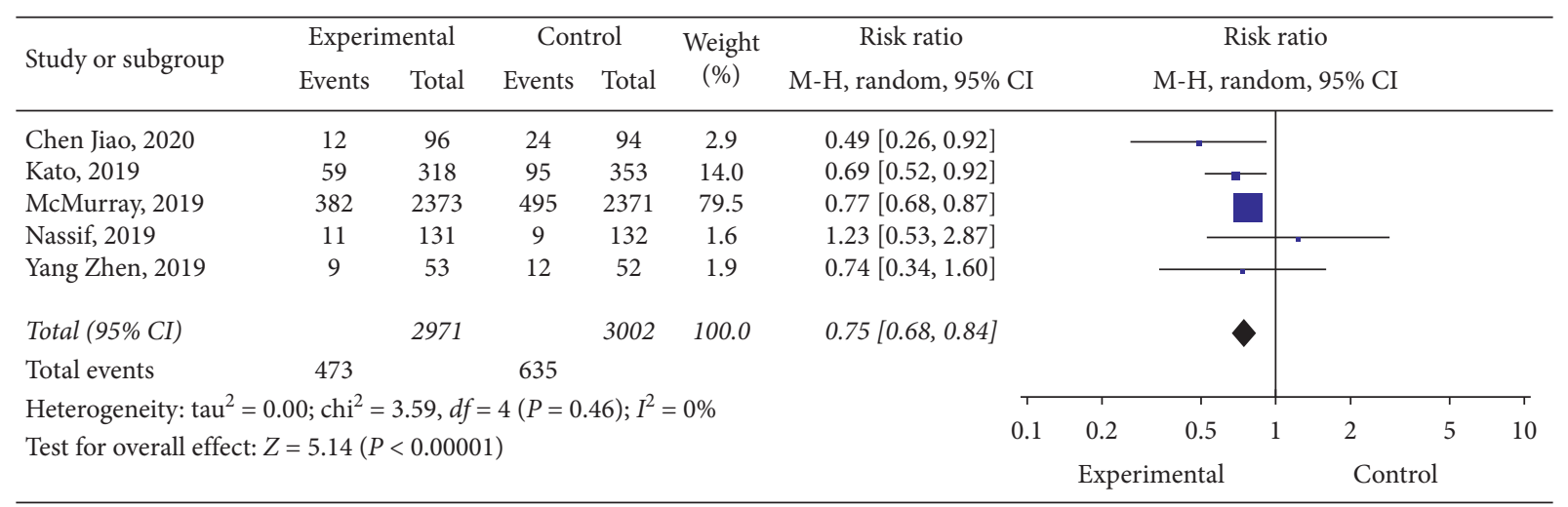

(a)

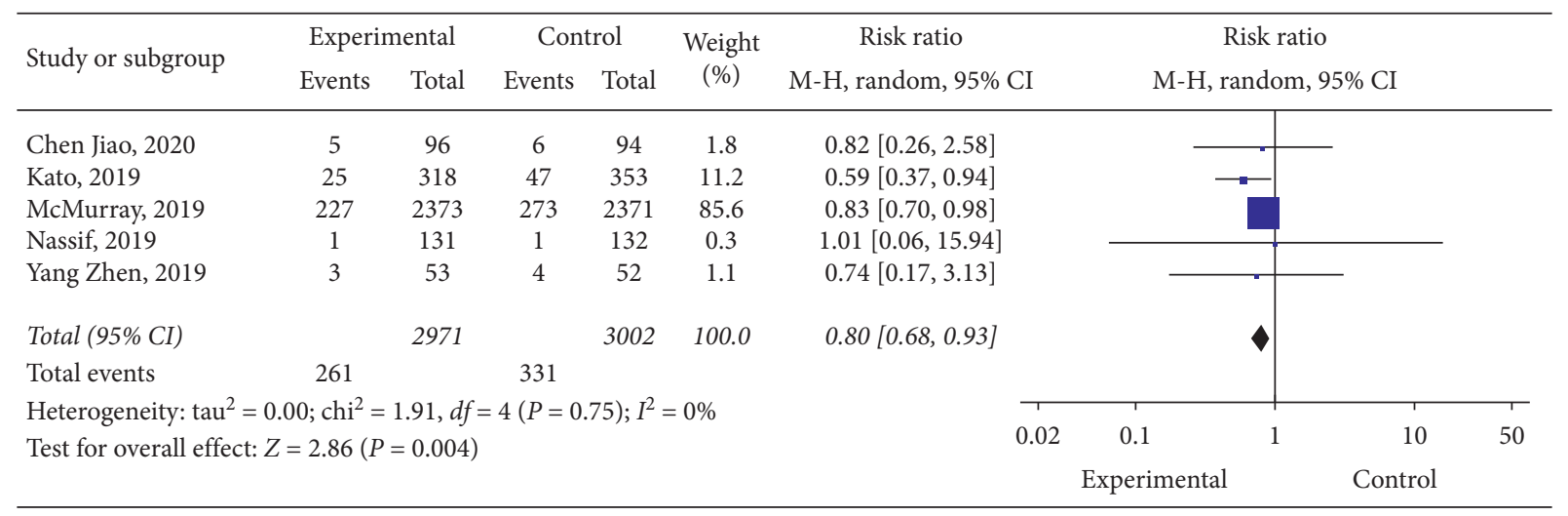

(b)

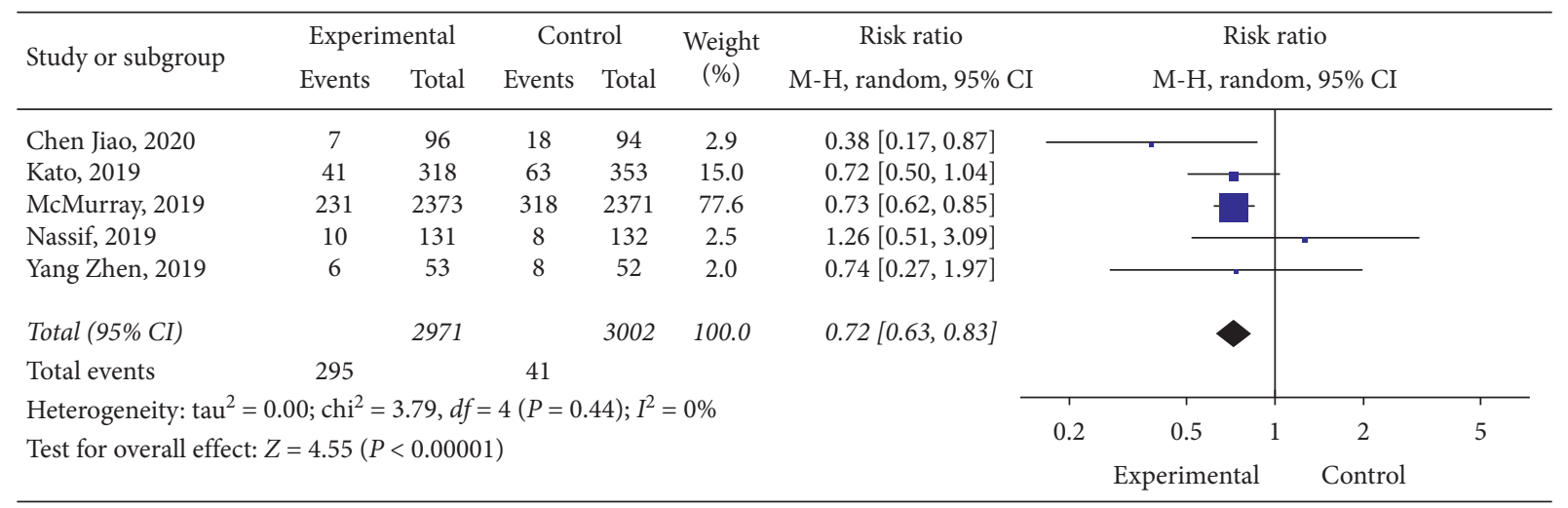

(c)

Figure 3: (a) Forest plot of the effect of dapagliflozin on cardiovascular death/hospitalization. (b) Forest plot of the effect of dapagliflozin on heart failure hospitalization. (c) Forest plot of the effect of dapagliflozin on cardiovascular death.

also has a positive effect on patients with heart failure [10-12, 21-23]. The FDA has recently approved dapagliflozin for adult patients with HFrEF (with or without T2DM) to decrease the danger of cardiovascular death and hospitalization for heart failure. However, its efficacy and clinical application are still controversial, and there are no standardized clinical guidelines for reference. Consequently, we thoroughly evaluated the literature to objectively appraise dapagliflozin.

The efficacy of dapagliflozin on the heart may be based on its ability to decrease preload of the heart by natriuresis and diuresis [24] and to decrease postload by reducing arterial blood pressure and changing vascular function [25]. SGLT2 inhibitors may also ameliorate and/or optimize cardiac energy metabolism and improve cardiac efficacy and cardiac output by increasing myocardial energy and substrate efficiency. Studies have shown that SGLT2 inhibitors may/could increase the production of ketone bodies and $\beta$-hydroxybutyrate. Cardiac uptake of carbohydrates (pyruvate, lactate, and glucose) was shown to be reduced, while uptake of the total $\beta$-hydroxybutyrate and ketone bodies was enhanced in diabetic patients when they were compared with nondiabetic patients. Consequently, ketone bodies may be used as a source of energy 


\begin{tabular}{|c|c|c|c|c|c|c|c|c|c|c|c|}
\hline \multirow{2}{*}{ Study or subgroup } & \multicolumn{2}{|c|}{ Experimental } & \multicolumn{2}{|c|}{ Control } & \multirow{2}{*}{$\begin{array}{l}\text { Weight } \\
(\%)\end{array}$} & \multirow{2}{*}{\multicolumn{2}{|c|}{$\begin{array}{c}\text { Risk ratio } \\
\mathrm{M}-\mathrm{H} \text {, random, 95\% CI }\end{array}$}} & \multirow{2}{*}{\multicolumn{3}{|c|}{$\begin{array}{c}\text { Risk ratio } \\
\text { M-H, random, } 95 \% \text { CI }\end{array}$}} & \\
\hline & Events & Total & Events & Total & & & & & & & \\
\hline Kato, 2019 & 7 & 318 & 10 & 353 & 55.3 & $0.78[0.30,2.02]$ & & & & & \\
\hline McMurray, 2019 & 4 & 2368 & 4 & 2368 & 26.2 & $1.00[0.25,3.99]$ & & & & & \\
\hline Nassif, 2019 & 1 & 131 & 1 & 132 & 6.6 & $1.01[0.06,15.94]$ & & & & & \\
\hline Yang Zhen, 2019 & 1 & 53 & 7 & 52 & 11.9 & $0.14[0.02,1.10]$ & & & & & \\
\hline Total $(95 \%$ CI) & & 2870 & & 2905 & 100.0 & $0.69[0.34,1.40]$ & & & & & \\
\hline Total events & 13 & & 22 & & & & & & & & \\
\hline \multicolumn{7}{|c|}{ Heterogeneity: $\operatorname{tau}^{2}=0.00 ; \mathrm{chi}^{2}=2.79, d f=3(P=0.42) ; I^{2}=0 \%$} & 0.005 & 0.1 & 1 & 10 & 200 \\
\hline \multicolumn{7}{|c|}{ Test for overall effect: $Z=1.03(P=0.30)$} & & Experime & & Control & \\
\hline
\end{tabular}

(a)

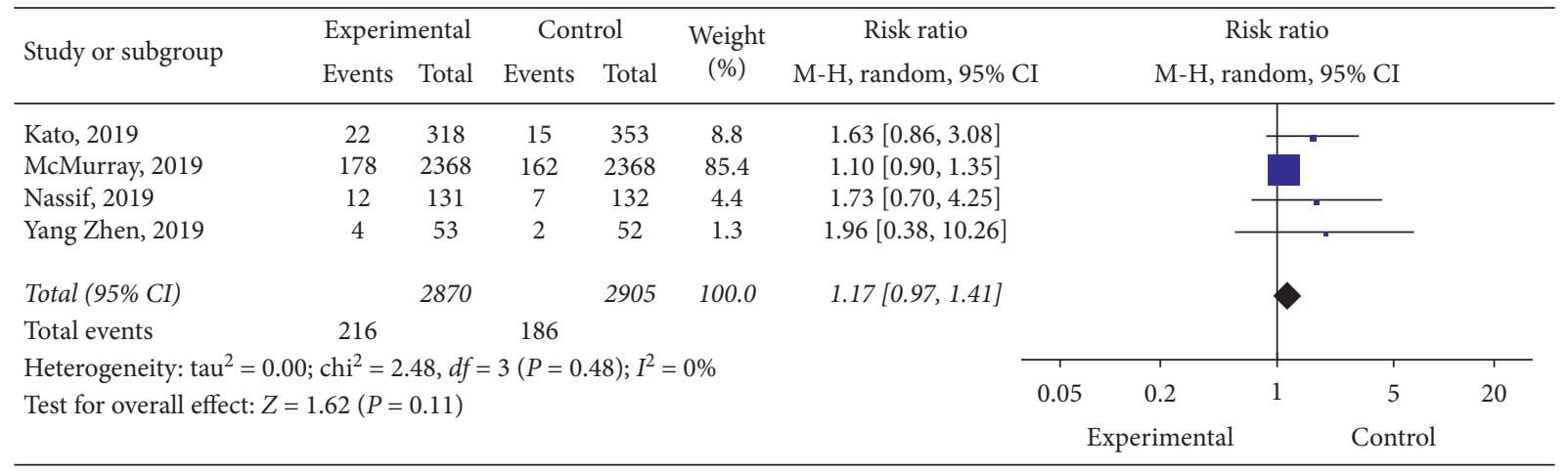

(b)

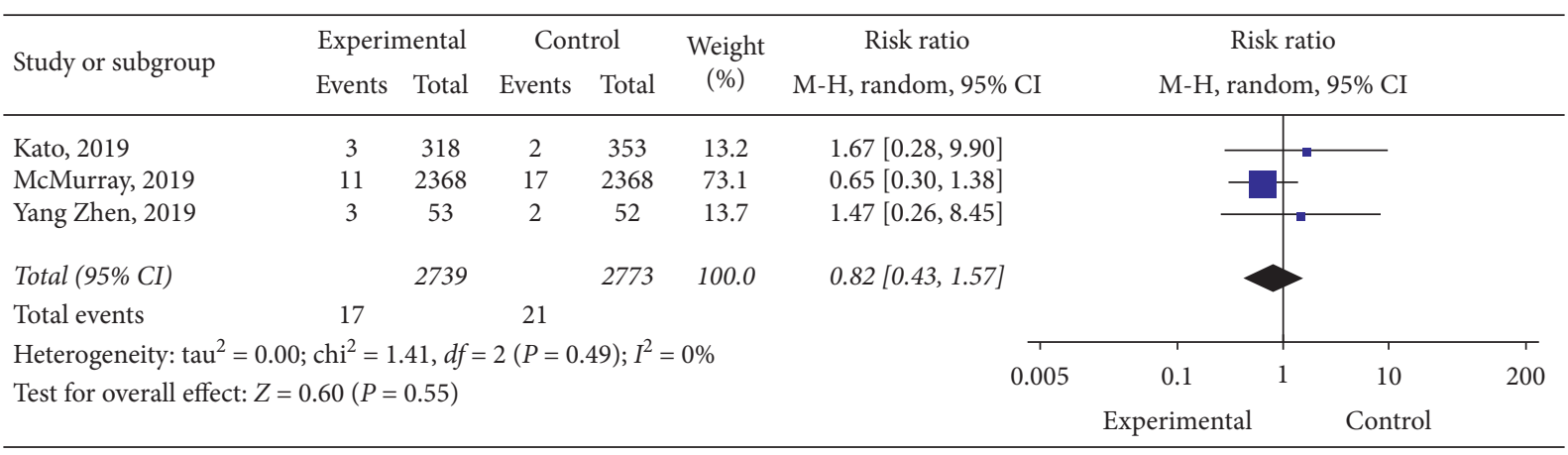

(c)

Figure 4: (a) Forest plot of the effect of dapagliflozin on hypoglycemia. (b) Forest plot of the effect of dapagliflozin on volume depletion. (c) Forest plot of the effect of dapagliflozin on urinary tract infection.

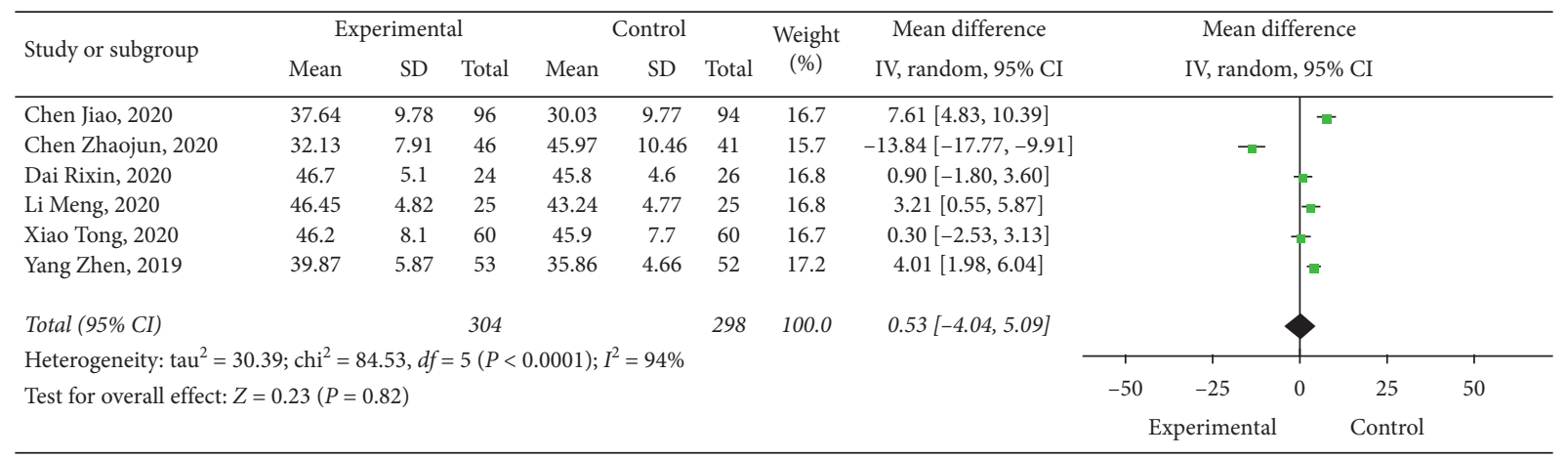

(a)

Figure 5: Continued. 


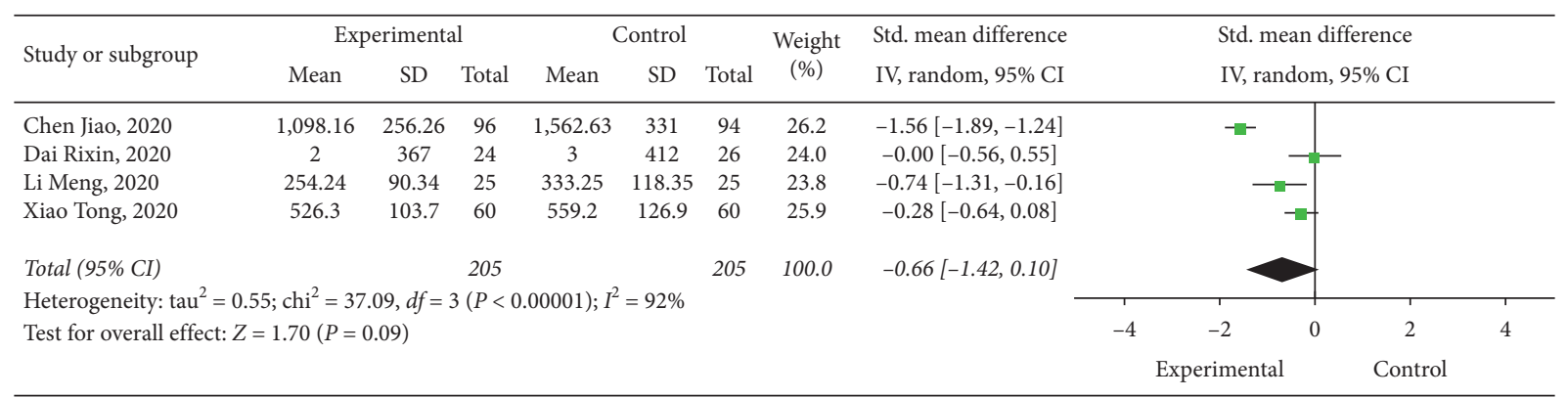

(b)

FIGURE 5: (a) Forest plot of the effect of dapagliflozin on left ventricular ejection fraction. (b) Forest plot of the effect of dapagliflozin on NTproBNP.

\begin{tabular}{|c|c|c|c|c|c|c|c|c|c|c|}
\hline \multirow{3}{*}{$\begin{array}{l}\text { Study or subgroup } \\
\text { 2.1.1 NYHA class II }\end{array}$} & \multicolumn{2}{|c|}{ Experimental } & \multicolumn{2}{|c|}{ Control } & \multirow{2}{*}{$\begin{array}{l}\text { Weight } \\
(\%)\end{array}$} & \multirow{2}{*}{\multicolumn{2}{|c|}{$\begin{array}{c}\text { Risk ratio } \\
\text { M-H, random, 95\% CI }\end{array}$}} & \multirow{2}{*}{\multicolumn{3}{|c|}{$\begin{array}{c}\text { Risk ratio } \\
\text { M-H, random, 95\% CI }\end{array}$}} \\
\hline & \multirow[t]{2}{*}{ Events } & \multirow[t]{2}{*}{ Total } & \multirow[t]{2}{*}{ Events } & \multirow[t]{2}{*}{ Total } & & & & & & \\
\hline & & & & & & & & & & \\
\hline Chen Jiao, 2020 & 69 & 100 & 64 & 100 & 2.8 & $1.08[0.89,1.31]$ & & & & \\
\hline Dai Rixin, 2020 & 19 & 24 & 20 & 26 & 1.3 & $1.03[0.77,1.38]$ & & & & \\
\hline Li Meng, 2020 & 9 & 25 & 8 & 25 & 0.2 & $1.13[0.52,2.44]$ & & & & \\
\hline McMurray, 2019 & 1606 & 2373 & 1597 & 2371 & 70.6 & $1.00[0.97,1.05]$ & & & & \\
\hline Nassif, 2019 & 91 & 131 & 82 & 132 & 3.6 & $1.12[0.94,1.33]$ & & & & \\
\hline Xiao Tong, 2020 & 38 & 60 & 42 & 160 & 1.7 & $0.90[0.70,1.17]$ & & & - & \\
\hline Yang Zhen, 2019 & 11 & 52 & 10 & 53 & 0.2 & $1.12[0.52,2.41]$ & & & & \\
\hline Subtotal (95\% CI) & & 2765 & & 2767 & 80.4 & $1.01[0.97,1.05]$ & & & & \\
\hline Total events & 1843 & & 1823 & & & & & & & \\
\hline Heterogeneity: $\operatorname{tau}^{2}=$ & $.00 ; \operatorname{chi}^{2}=$ & $=2.67, d$ & $f=6(P=$ & $0.85) ;$ & $=0 \%$ & & & & & \\
\hline Test for overall effect & $Z=0.57(1$ & $=0.57$ & & & & & & & & \\
\hline 2.1.2 NYHA class III & & & & & & & & & & \\
\hline Chen Jiao, 2020 & 28 & 100 & 32 & 100 & 0.6 & $0.88[0.57,1.34]$ & & & & \\
\hline Dai Rixin, 2020 & 5 & 24 & 5 & 26 & 0.1 & $1.08[0.36,3.28]$ & 4 & & & $\rightarrow$ \\
\hline Li Meng, 2020 & 12 & 25 & 12 & 25 & 0.3 & $1.00[0.56,1.78]$ & & & & \\
\hline McMurray, 2019 & 747 & 2373 & 751 & 2371 & 15.7 & $0.99[0.91,1.08]$ & & & 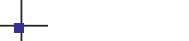 & \\
\hline Nassif, 2019 & 40 & 131 & 50 & 132 & 1.0 & $0.81[0.57,1.13]$ & & & & \\
\hline Xiao Tong, 2020 & 18 & 60 & 16 & 60 & 0.3 & $1.13[0.64,1.99]$ & & & & \\
\hline Yang Zhen, 2019 & 28 & 52 & 28 & 53 & 0.9 & $1.02[0.71,1.46]$ & & & & \\
\hline Subtotal (95\% CI) & & 2765 & & 2767 & 18.9 & $0.98[0.91,1.06]$ & & & & \\
\hline Total events & 878 & & 894 & & & & & & & \\
\hline Heterogeneity: tau $^{2}=$ & $.00 ; \mathrm{chi}^{2}$ & $=1.96, d$ & $f=6(P=$ & $0.92) ;$ & $=0 \%$ & & & & & \\
\hline Test for overall effect & $Z=0.44(1$ & $=0.66$ & & & & & & & & \\
\hline 2.1.3 NYHA class IV & & & & & & & & & & \\
\hline Chen Jiao, 2020 & 3 & 100 & 4 & 100 & 0.1 & $0.75[0.17,3.27]$ & 4 & & & $\rightarrow$ \\
\hline Li Meng, 2020 & 4 & 24 & 5 & 26 & 0.1 & $0.80[0.24,2.64]$ & $\longleftarrow$ & & & \\
\hline McMurray, 2019 & 20 & 2373 & 23 & 2371 & 0.3 & $0.87[0.48,1.58]$ & - & & & \\
\hline Xiao Tong, 2020 & 4 & 60 & 2 & 601 & 0.0 & $2.00[0.38,10.51]$ & $\leftarrow$ & & & $\rightarrow$ \\
\hline Yang Zhen, 2019 & 14 & 52 & 15 & 53 & 0.3 & $0.95[0.51,1.77]$ & & & & \\
\hline Subtotal (95\% CI) & & 2610 & & 2609 & 0.8 & $0.92[0.63,1.35]$ & & & & \\
\hline Total events & 45 & & 49 & & & & & & & \\
\hline Heterogeneity: $\operatorname{tau}^{2}=$ & $.00 ; \operatorname{chi}^{2}$ & $=1.01, d$ & $f=4(P=$ & $0.91) ;$ & $=0 \%$ & & & & & \\
\hline Test for overall effect & $=0.42$ & $P=0.68$ & & & & & & & & \\
\hline Total (95\% CI) & & 8140 & & 8143 & 100.0 & $1.00[0.97,1.04]$ & & & 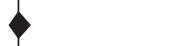 & \\
\hline Total events & 2766 & & 2766 & & & & & & & \\
\hline Heterogeneity: tau $^{2}=$ & $.00 ; \operatorname{chi}^{2}$ & $=6.31, d$ & $f=18(P$ & $=0.99)$; & $I^{2}=0 \%$ & & 0.5 & 0.7 & 1.5 & 2 \\
\hline $\begin{array}{l}\text { Test for overall effect } \\
\text { Test for subgroup dif }\end{array}$ & $\begin{array}{l}Z=0.28(1 \\
\text { rences } \cdot \mathrm{ch}\end{array}$ & $\begin{array}{l}P=0.78 \\
i^{2}=0.6\end{array}$ & $d f=2($ & $=0.7$ & $I^{2}=0 \%$ & & & Experimental & Control & \\
\hline
\end{tabular}

FIgURE 6: Subgroup analysis about the effect of heart function on results. 


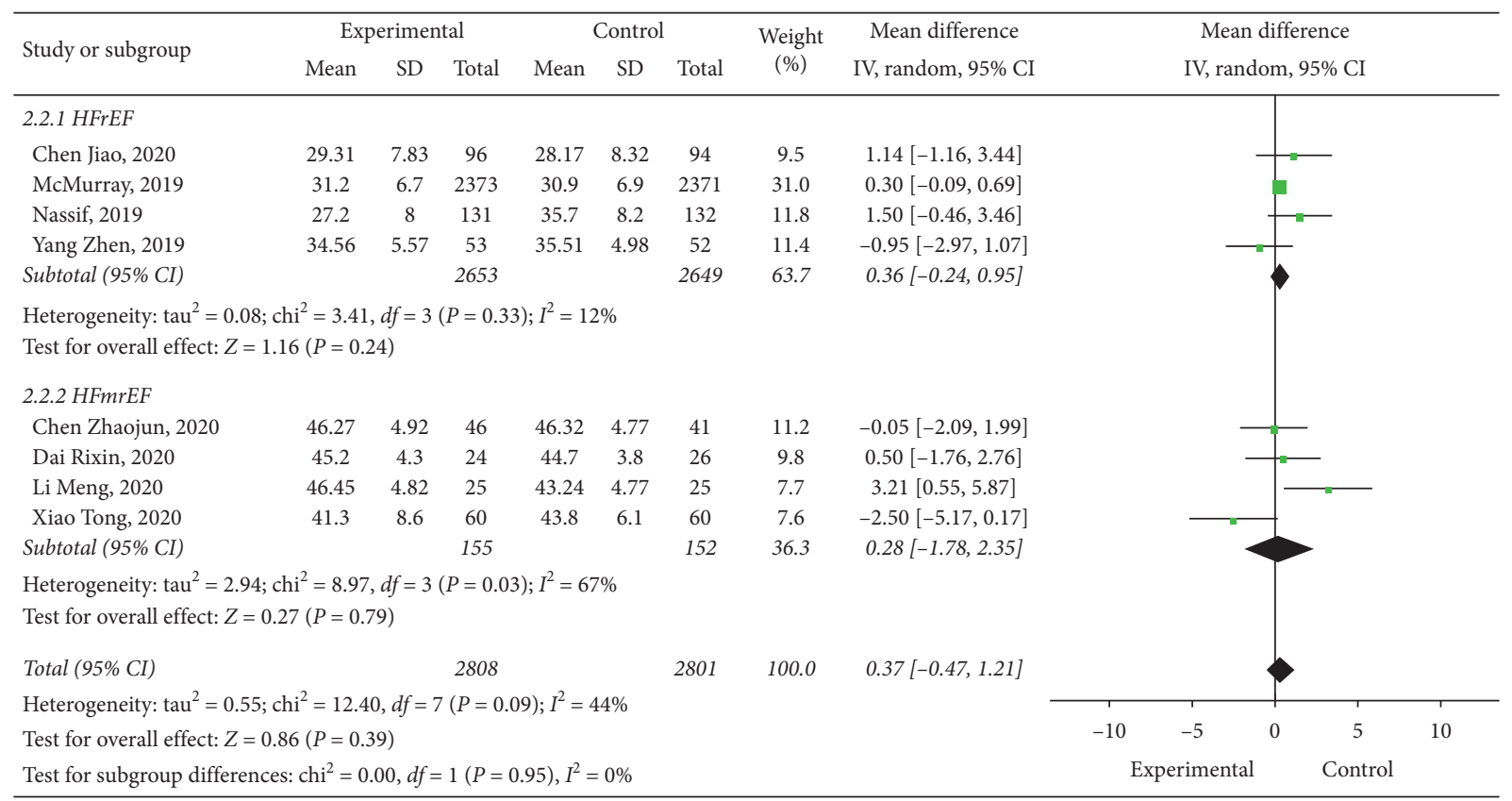

Figure 7: Subgroup analysis about HFrEF and HFmrEF.

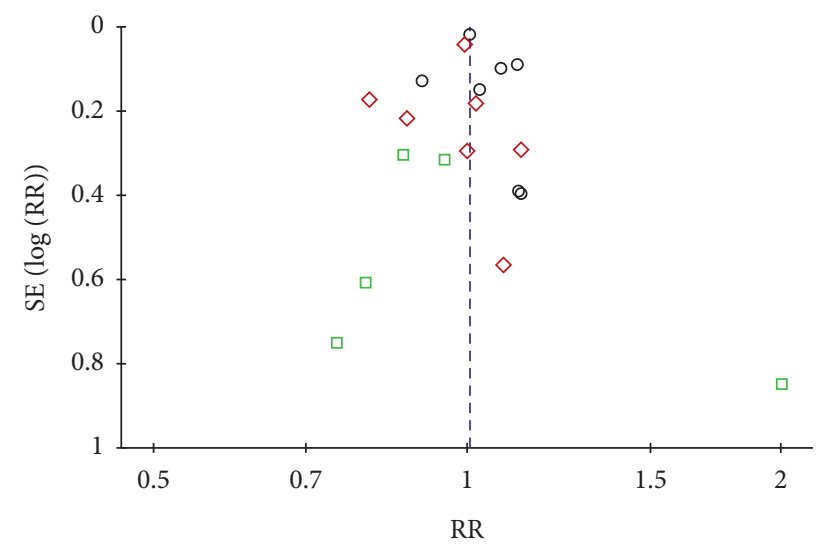

$$
\begin{aligned}
& \text { Subgroups } \\
& \bigcirc \text { NYHA class II } \\
& \diamond \text { NYHA class III } \\
& \square \text { NYHA class IV }
\end{aligned}
$$

Figure 8: Funnel plot of subgroup analysis about the effect of heart function on results.

moderately substituting glucose in patients with diabetes [26-28]. This theory is supported by animal experiments, indicating that SGLT2 inhibitors may/could increase myocardial ketone body consumption and reduce cardiac glucose utilization and lactate production [29]. However, an in-depth analysis is required to confirm this possibility. At the same time, studies have found that SGLT2 inhibition can promote the degradation of branched-chain amino acids, thereby providing an energy source for myocardium in patients with T2DM and HF [30]. SGLT2 inhibitors are anti-inflammatory and have an inhibitory effect on cardiac fibrosis. Myocardial fibrosis is widely considered to be an important factor in the development of HF. Recently, some experimental data indicate that SGLT2 inhibits collagen fiber synthesis by augmenting the activation of M2 macrophages and restraining myofibroblast differentiation, thus showing significant antifibrosis effect [31].

In this meta-analysis, we quantitatively analyzed the efficacy and safety of dapagliflozin for the management of chronic heart failure. We showed that dapagliflozin can significantly decrease the frequency of cardiovascular death/hospitalization for heart failure, cardiovascular death, and hospitalization for heart failure. Furthermore, it 
improves survival and reduces the need for hospitalization. This suggests that dapagliflozin may provide an extra treatment option for patients with chronic heart failure in addition to ACEIs, ARNIs, beta-blockers, and MRAs. In summary, patients with HF should be treated with a combination of the following four drugs as early as possible: an ARNI, beta-blocker, MRA, and SGLT2 inhibitor. Volume depletion, hypoglycemia, and urinary tract infections are not affected by dapagliflozin. In the past, it was believed that patients with poorer heart function and left ventricular ejection fraction had a worse prognosis. However, subgroup analysis showed surprising results: the effect of dapagliflozin was not affected by heart function grades and LVEF. The positive effect of dapagliflozin in heart failure has nothing to do with the degree of heart function and LVEF. Therefore, dapagliflozin treatment is applicable in patients with mild and severe HF. Besides, dapagliflozin decreased the risk of death and deteriorating the $\mathrm{HF}$ and relieved symptoms among different ages of people [21]. Some researchers believe that the combination of new targeted drugs such as aldosterone receptor antagonists, angiotensin receptor-neprilysin inhibitors, and SGLT2 inhibitors could significantly decrease the risk of death in patients with heart failure. Some studies have also shown that dapagliflozin has a positive effect on blood pressure reduction, weight loss, left ventricular remodeling, reducing acute kidney injury, atrial fibrillation, and atrial flutter $[15,16]$. The findings of our meta-analysis suggest that dapagliflozin has useful effects other than glucose lowering [9, 32-37]; thus, they potentially expand the therapeutic role of dapagliflozin beyond patients with diabetes.

Although dapagliflozin treatment carries several advantages, it also leads to some adverse reactions in clinical practice. Diabetic ketoacidosis (DKA) is a common complication in patients with diabetes, which is more common in patients with T1D than T2D patients. Several SGLT2 inhibitors have been FDA approved for the management of T1DM in the EU and Japan, but they have not yet been approved in the United States and China. In March and July 2019, respectively, sotagliflozin and dapagliflozin were rejected by the FDA for the treatment of T1DM, and in March 2020, empagliflozin was rejected because a higher number of patients in the experimental group developed DKA compared with that of the placebo group [38, 39]. Besides, some studies showed that dapagliflozin increased the incidence of rare and chronic infectivity of the genitals and surrounding areas, called as Fournier's gangrene (FG), which is a life-threatening fulminant infection that affects the subcutaneous tissue around the genitals. The disease is characterized by rapid onset and hyperthermia, extensive necrosis, and gangrene in the infected tissue $[40,41]$.

\section{Limitations}

This study has some limitations. The population that met the inclusion criteria was HFrEF and HFmrEF patients, and patients with preserved ejection fraction heart failure
(HFpEF) may not be eligible for this drug. In this study, males accounted for $77 \%$ of the population, and black patients accounted for only 5.7\%, which might affect the generalizability of the study data. However, to obtain further robust evidence to guide clinical practice, more extensive, multicenter, and high-quality clinical research must be performed.

\section{Conclusion}

We assessed the incidence of cardiovascular death/hospitalization for heart failure, cardiovascular death, and hospitalization for heart failure in patients with heart failure. Dapagliflozin significantly decreased the incidence of $\mathrm{CV}$ death/HHF (relative risk (RR): 0.75; 95\% confidence interval (CI): 0.68-0.84), CV death (RR: 0.80; 95\% CI: 0.68-0.93), and HHF (RR: 0.72; 95\% CI: 0.63-0.83).

\section{Abbreviations}

SGLT2: Sodium-glucose cotransporter-2

CHF: $\quad$ Chronic heart failure

NCKI: China National Knowledge Infrastructure

CV death: Cardiovascular death

HHF: Hospitalization for heart failure

LVEF: Left ventricular ejection fraction

NT- N-terminal pro-B-type natriuretic peptide

proBNP:

RR: $\quad$ Relative risk

CI: $\quad$ Confidence interval

T2DM: $\quad$ Type 2 diabetes mellitus

FDA: $\quad$ Food and Drug Administration

HF: Heart failure

HFrEF: $\quad$ Heart failure and reduced ejection fraction

RCTs: $\quad$ Randomized controlled trials

NYHA: New York Heart Association

eGFR: $\quad$ Estimated glomerular filtration rate

DKA: Diabetic ketoacidosis

T1DM: $\quad$ Type 1 diabetes mellitus

FG: $\quad$ Fournier's gangrene

HFmrEF: Midrange ejection fraction heart failure

HFpEF: Heart failure and preserved ejection fraction

DAPA: Dapagliflozin

RT: $\quad$ Recommended therapy

SD: $\quad$ Standard deviation

ACEIs: Angiotensin-converting enzyme inhibitors

ARNIs: Angiotensin receptor/neprilysin inhibitors

MRAs: $\quad$ Mineralocorticoid receptor antagonists.

\section{Data Availability}

1. The datasets generated and/or analyzed during the current study are available in the PubMed, Cochrane Library, Embase, NCKI, VIP, Wanfang Data, and ClinicalTrials.gov repository (https://pubmed.ncbi.nlm.nih.gov/, https://www.cochranelibrary.com/, https://www.embase. com/, https://www.cnki.net/, http://www.cqvip.com/, http://www.wanfangdata.com.cn/index.html, and https:// www.clinicaltrials.gov/). 2. The datasets generated and/or 
analyzed during the current study are available from the corresponding author upon reasonable request. 3. All the data generated or analyzed during this study are included within this published article (and its supplementary information files).

\section{Conflicts of Interest}

The authors declare that they have no conflicts of interest.

\section{Authors' Contributions}

R. P. C. made substantial contributions to conception and design of the study and wrote the manuscript; R. P. C. and Y. L. X. searched the literature, extracted the data from the collected literature, and analyzed the data; and Q. S. revised the manuscript. All authors approved the final version of the manuscript.

\section{Supplementary Materials}

Supplementary: PRISMA 2009 Checklist. (Supplementary Materials)

\section{References}

[1] P. Ponikowski, A. A. Voors, S. D. Anker et al., "2016 ESC Guidelines for the diagnosis and treatment of acute and chronic heart failure," European Heart Journal, vol. 37, no. 27, pp. 2129-2200, 2016.

[2] GBD 2016 Disease and Injury Incidence and Prevalence Collaborators, "Global, regional, and national incidence, prevalence, and years lived with disability for 328 diseases and injuries for 195 countries, 1990-2016: a systematic analysis for the Global Burden of Disease Study 2016," Lancet, vol. 390, no. 10100, pp. 1211-1259, 2017.

[3] J. E. Udelson and L. W. Stevenson, "The future of heart failure diagnosis, therapy, and management," Circulation, vol. 133, no. 25, pp. 2671-2686, 2016.

[4] D. Fitchett, S. E. Inzucchi, C. P. Cannon et al., "Empagliflozin reduced mortality and hospitalization for heart failure across the spectrum of cardiovascular risk in the EMPA-REG OUTCOME trial," Circulation, vol. 139, no. 11, pp. 1384-1395, 2019.

[5] B. Zinman, C. Wanner, J. M. Lachin et al., "Empagliflozin, cardiovascular outcomes, and mortality in type 2 diabetes," New England Journal of Medicine, vol. 373, no. 22, pp. 2117-2128, 2015.

[6] V. Perkovic, M. J. Jardine, B. Neal et al., "Canagliflozin and renal outcomes in type 2 diabetes and nephropathy," New England Journal of Medicine, vol. 380, no. 24, pp. 2295-2306, 2019.

[7] B. Neal, V. Perkovic, K. W. Mahaffey et al., "Canagliflozin and cardiovascular and renal events in type 2 diabetes," New England Journal of Medicine, vol. 377, no. 7, pp. 644-657, 2017.

[8] S. D. Wiviott, I. Raz, M. P. Bonaca et al., "Dapagliflozin and cardiovascular outcomes in type 2 diabetes," New England Journal of Medicine, vol. 380, no. 4, pp. 347-357, 2019.

[9] T. A. Zelniker, S. D. Wiviott, I. Raz et al., "SGLT2 inhibitors for primary and secondary prevention of cardiovascular and renal outcomes in type 2 diabetes: a systematic review and meta-analysis of cardiovascular outcome trials," The Lancet, vol. 393, no. 10166, pp. 31-39, 2019.

[10] J. J. V. McMurray, S. D. Solomon, S. E. Inzucchi et al., "Dapagliflozin in patients with heart failure and reduced ejection fraction," The New England Journal of Medicine, vol. 381, no. 21, pp. 1995-2008, 2019.

[11] M. E. Nassif, S. L. Windsor, F Tang et al., "Dapagliflozin effects on biomarkers, symptoms, and functional status in patients with heart failure with reduced ejection fraction: the DEFINEHF trial," Circulation, vol. 140, no. 18, pp. 1463-1476, 2019.

[12] E. T. Kato, M. G. Silverman, O. Mosenzon et al., "Effect of dapagliflozin on heart failure and mortality in type 2 diabetes mellitus," Circulation, vol. 139, no. 22, pp. 2528-2536, 2019.

[13] J. Chen, J. Zhou, B. Tang et al., "Therapeutic effect of dapagliflozin on patients with diabetes mellitus complicated HF and its influence on vascular endothelial function and inflammatory factor levels," Chinese Journal of Cardiovascular Rehabilitation Medicine, vol. 29, no. 5, pp. 599-603, 2020.

[14] Z. Chen and H. Jiang, "Analysis of the effect of dapagliflozin on HbAlc level and heart function in patients with diabetes mellitus complicated with chronic heart failure," Journal of Aerospace Medicine, vol. 31, no. 11, pp. 1350-1351, 2020.

[15] R. Dai, L. Liu, X. Yang et al., "Effects of Dapagliflozin on midrange ejection fraction heart failure patients with type 2 diabetes," The Journal of Practical Medicine, vol. 36, no. 18, pp. 2505-2509, 2020.

[16] Li Meng, "Clinical study of metformin combined with dapagliflozin in the treatment of type 2 diabetes patients with heart failure," Journal of North Pharmacy, vol. 17, no. 4, pp. 124-125, 2020.

[17] X. Tong, Y. Liu, J. Chen et al., "Application of dapagliflozin in treatment of elderly CHF patients with type $2 \mathrm{DM}$ and hyperuricemia," vol. 22, no. 10, pp. 1016-1019, 2020.

[18] Z. Yang, J. Dong, J. Zhang et al., "Clinical evaluation and analysis of dapagliflozin in patients with type 2 diabetes and cardiac insufficiency," Chongqing Medicine, vol. 48, no. 16, pp. 2861-2863, 2019.

[19] J. P. T. Higgins, D. G. Altman, and J. A. C. Sterne, "Chapter 8: assessing risk of bias in included studies," in Cochrane Handbook for Systematic Reviews of Interventions Version 5.1.0, J. P. T. Higgins and S. Green, Eds., The Cochrane Collaboration, London, Uk, 2011.

[20] H. Yaribeygi, T. Sathyapalan, M. Maleki, T. Jamialahmadi, and A. Sahebkar, "Molecular mechanisms by which SGLT2 inhibitors can induce insulin sensitivity in diabetic milieu: a mechanistic review," Life Science, vol. 240, Article ID 117090, 2020.

[21] F. A. Martinez, M. Serenelli, J. C. Nicolau et al., "Efficacy and safety of dapagliflozin in heart failure with reduced ejection fraction according to age," Circulation, vol. 141, no. 2, pp. $100-111,2020$.

[22] M. C. Petrie, S. Verma, K. F. Docherty et al., "Effect of dapagliflozin on worsening heart failure and cardiovascular death in patients with heart failure with and without diabetes," JAMA, vol. 323, no. 14, p. 1353, 2020.

[23] M. N. Kosiborod, P. S. Jhund, K. F. Docherty et al., "Effects of dapagliflozin on symptoms, function, and quality of life in patients with heart failure and reduced ejection fraction," Circulation, vol. 141, no. 2, pp. 90-99, 2020.

[24] S. Verma, J. J. V. McMurray, and D. Z. I. Cherney, "The metabolodiuretic promise of sodium-dependent glucose cotransporter 2 inhibition," JAMA Cardiology, vol. 2, no. 9, pp. 939-940, 2017. 
[25] H. Sternlicht and G. L. Bakris, "Blood pressure lowering and sodium-glucose Co-transporter 2 inhibitors (SGLT2is): more than osmotic diuresis," Current Hypertension Reports, vol. 21, no. 2, p. 12, 2019.

[26] E. Ferrannini, M. Mark, and E. Mayoux, "CV protection in the EMPA-REG outcome trial: a "thrifty substrate" hypothesis," Diabetes Care, vol. 39, no. 7, pp. 1108-1114, 2016.

[27] Y. Mizuno, E. Harada, H. Nakagawa et al., "The diabetic heart utilizes ketone bodies as an energy source," Metabolism, vol. 77, pp. 65-72, 2017.

[28] L. C. Gormsen, M. Svart, H. H. Thomsen et al., "Ketone body infusion with 3-hydroxybutyrate reduces myocardial glucose uptake and increases blood flow in humans: a positron emission tomography study," Journal of the American Heart Association, vol. 6, no. 3, Article ID e005066, 2017.

[29] H. Oshima, T. Miki, A. Kuno et al., "Empagliflozin, an SGLT2 inhibitor, reduced the mortality rate after acute myocardial infarction with modification of cardiac metabolomes and antioxidants in diabetic rats," Journal of Pharmacology and Experimental Therapeutics, vol. 368, no. 3, pp. 524-534, 2019.

[30] B. A. Kappel, M. Lehrke, K. Schütt et al., "Effect of empagliflozin on the metabolic signature of patients with type 2 diabetes mellitus and cardiovascular disease," Circulation, vol. 136, no. 10, pp. 969-972, 2017.

[31] T.-M. Lee, N.-C. Chang, and S.-Z. Lin, "Dapagliflozin, a selective SGLT2 Inhibitor, attenuated cardiac fibrosis by regulating the macrophage polarization via STAT3 signaling in infarcted rat hearts," Free Radical Biology and Medicine, vol. 104, pp. 298-310, 2017.

[32] M. Packer, S. D. Anker, J. Butler, G. Filippatos, and F. Zannad, "Effects of sodium-glucose cotransporter 2 inhibitors for the treatment of patients with heart failure," JAMA Cardiology, vol. 2, no. 9, pp. 1025-1029, 2017.

[33] S. Verma and J. J. V. McMurray, "SGLT2 inhibitors and mechanisms of cardiovascular benefit: a state-of-the-art review," Diabetologia, vol. 61, no. 10, pp. 2108-2117, 2018.

[34] S. E. Inzucchi, M. Kosiborod, D. Fitchett et al., "Improvement in cardiovascular outcomes with empagliflozin is independent of glycemic control," Circulation, vol. 138, no. 17, pp. 1904-1907, 2018.

[35] Y. Lytvyn, P. Bjornstad, J. A. Udell, J. A. Lovshin, and D. Z. I. Cherney, "Sodium glucose cotransporter-2 inhibition in heart failure," Circulation, vol. 136, no. 17, pp. 1643-1658, 2017.

[36] F. Bonnet and A. J. Scheen, "Effects of SGLT2 inhibitors on systemic and tissue low-grade inflammation: the potential contribution to diabetes complications and cardiovascular disease," Diabetes \& Metabolism, vol. 44, no. 6, pp. 457-464, 2018.

[37] C. Wanner, S. E. Inzucchi, J. M. Lachin et al., "Empagliflozin and progression of kidney disease in type 2 diabetes," New England Journal of Medicine, vol. 375, no. 4, pp. 323-334, 2016.

[38] US FDA Issues Complete Response Letter for Empagliflozin $2.5 \mathrm{Mg}$ as Adjunct to Insulin for Adults with Type 1 Diabetes.

[39] M. Fralick, S. Schneeweiss, and E. Patorno, "Risk of diabetic ketoacidosis after initiation of an SGLT2 inhibitor," New England Journal of Medicine, vol. 376, no. 23, pp. 2300-2302, 2017.

[40] C. E. Onder, K. Gursoy, S. M. Kuskonmaz, U. Kocer, and C. Culha, "Fournier's gangrene in a patient on dapagliflozin treatment for type 2 diabetes," Journal of Diabetes, vol. 11, no. 5, pp. 348-350, 2019.
[41] P. Ueda, H. Svanström, M. Melbye et al., "Sodium glucose cotransporter 2 inhibitors and risk of serious adverse events: nationwide register based cohort study," BMJ, vol. 363, p. k4365, 2018. 